\title{
A TRANSFORMATION WITH SIMPLE SPECTRUM WHICH IS NOT RANK ONE
}

\author{
ANDRÉS DEL JUNCO
}

Introduction. Following [10] an ergodic measure-preserving transformation is called rank one if it admits a sequence of approximating stacks. Rank one transformations have been studied in $[\mathbf{1}]$ and [2] where it was shown that any rank one transformation has simple spectrum. More generally it has been shown by Chacon [4] that a transformation of rank $n$ has spectral multiplicity at most $n$. M. A. Akcoglu and J. R. Baxter have asked whether the converse is true. In particular: does simple spectrum imply rank one? In this paper we give a negative answer to this question. The counterexample used is a transformation $\tau$ constructed by Baxter in [2] using double stacks. It was already observed in [2] that $\tau$ has simple spectrum. Our proof that $\tau$ is not of rank one depends on a number of arguments of a combinatorial nature. At the core of these is a combinatorial lemma about the Morse minimal sequence which may be of independent interest.

In passing, we establish that $\tau$ is (measure theoretically) identical with the dynamical system $\sigma$ generated by the Morse minimal sequence, which has been studied in a number of papers, notably [8] and [9], where a fairly detailed spectral analysis of $\sigma$ may be found. However, the proof that $\tau$ (or $\sigma$ ) has simple spectrum does not appear explicitly in $[\mathbf{2}],[\mathbf{8}]$ or $[\mathbf{9}]$, so we give it here. It seems that stacking constructions can be given for many other known examples of dynamical systems arising from uniquely ergodic sequences and this will be considered in a future paper. I am grateful to Professor Kakutani for several valuable conversations which brought to light the connection between his work, which was previously unknown to me, and Baxter's example.

We conclude by giving a characterization of rank one transformations which implies that factors of rank one transformations have rank one. This result is easily generalized to rank $n$ as well. (It has recently been drawn to my attention that the result on factors is stated without proof in $[\mathbf{1 0}]$ ). It should be pointed out that a factor of a transformation with simple spectrum again has simple spectrum so that if the result on factors of rank one were not true one would automatically have a simple spectrum transformation which is not rank one.

1. Definitions and notation. A transformation of a probability space $(X, \mathscr{F}, \mu)$ is a $1-1$ bimeasurable mapping $\tau: X \rightarrow X$ which preserves $\mu$. $(X, \mathscr{F}, \mu)$ is always assumed to be isomorphic to the unit interval with Borel

Received August 4, 1976 and in revised form, December 7, 1976. 
sets and Lebesgue measure. A semi-partition of $X$ is a finite collection $\left\{Q_{\gamma}: \gamma \in \Gamma\right\}$ of disjoint sets in $X$. We write $|Q|=\bigcup\left\{Q_{\gamma}: \gamma \in \Gamma\right\}$. If $|Q|=X$, $Q$ is called simply a partition.

If $Q$ is a partition the $Q$ - $n$-name of $x \in X$ is the $n$-tuple $(\xi(0), \ldots, \xi(n-1))$ $\in \Gamma^{n}$ defined by $\tau^{i} x \in Q_{\xi(i)}, 0 \leqq i \leqq n-1$. The pair $(\tau, Q)$ induces a measure on each $\Gamma^{n}$ denoted by $P$ and defined by

$$
P(\xi)=\mu\{x: Q-n \text {-name of } x \text { is } \xi\} \text {. }
$$

$P$ extends to a shift invariant measure on $\Gamma^{z}$ so $(\tau, Q)$ induces a stationary process $\{\xi(i)\}$, where $\xi(i)$ is the $i$ th coordinate function on $\Gamma^{2}$. We shall also refer to $(\tau, Q)$ itself as a process. When we speak of the probability of a set of $n$-tuples in $\Gamma^{n}$ this is understood to refer to $P$.

We denote by $Q_{m}{ }^{n}$ the partition $\bigvee_{i=m}^{n} \tau^{i} Q \cdot Q_{-\infty}^{\infty}$ denotes the $\sigma$-field generated by the $\tau^{i} Q, i \in Z$. Note that $Q_{-(n-1)}^{0}$ is the partition of $X$ according to $Q-n$ names.

If $Q$ and $R$ are two semi-partitions we write $Q \subset R$ if $|Q|=|R|$ and each atom of $Q$ is a union of atoms of $R$. If $Q$ and $R$ are semi-partitions both indexed by $\Gamma$ we write $D(Q, R)=\bigcup_{\gamma \in \Gamma}\left(Q_{\gamma} \Delta R_{\gamma}\right)$. For $E \in \mathscr{F}, \rho_{E}(Q, R)=\mu_{E}(D)=$ $\mu(E)^{-1} \mu(D \cap E) . Q \cap E$ denotes the semi-partition $\left\{Q_{\gamma} \cap E: \gamma \in \Gamma\right\}$. Note that $\rho(Q \cap E, R \cap E) \neq \rho_{E}(Q, R)$ as we do not condition $\mu$ to find $\rho(Q \cap E$, $R \cap E)$.

If $\Gamma$ is any finite index set and $\xi \in \Gamma^{n}$ we refer to $\xi$ as an $n$-string and denote its length $n$ by $|\xi|$. If $0 \leqq i \leqq j \leqq n-1, \xi[i, j]$ denotes the $(j-i+1)$-string $(\xi(i), \ldots, \xi(j))$. If $\xi$ and $\eta$ are $n$-strings we set

$$
d(\xi, \eta)=n^{-1} \#\{i: \xi(i) \neq \eta(i)\} .
$$

If $d(\xi, \eta) \leqq \epsilon$ we say $\xi$ equals (or agrees with or is) $\eta$ within $\epsilon$.

A $\tau$-stack $S$ is a semi-partition $\left\{S_{0}, \ldots, S_{n-1}\right\}$ of $X$ such that $\tau S_{i}=S_{i+1}$ for $0 \leqq i<n-1 . S_{0}$ is called the base of $S$ (written $B(S), S_{n-1}$ the top and $n$ the height. Following [10] we say $\tau$ has rank at most $n$ if for each partition $Q$ and $\epsilon>0$ there are semi-partitions $Q^{\prime}$ and $R$ such that $Q^{\prime} \subset R, \rho\left(Q, Q^{\prime}\right)<\epsilon$ and $Q^{\prime}$ can be split into a disjoint union of $n \tau$-stacks. The rank of $\tau$ is the least integer $n$ (possibly infinite) such that $\tau$ has rank at most $n$. It has been shown by Baxter $[\mathbf{1}]$ that if $\tau$ has rank one then $\tau$ can be constructed by the stacking method using single stacks (see [5]). Baxter's argument may be used to show that a transformation with rank $n$ can be constructed by the stacking method with $n$ stacks. Thus the class of finite rank transformations is completely concrete. Note that any rank one transformation is necessarily ergodic, but that is not so for rank $n$.

2. Although we shall give an exact characterization of rank one in Section 3, in this section we will need the following necessary condition for rank one to show that our example does not have rank one. It would be interesting to know if this condition is sufficient as well. 
Proposition 2.1. If $(T, Q)$ is a process such that $T$ has rank one and $\delta>0$ then for infinitely many $n$ there is an $n$-string $\xi_{0}$ of positive probability such that

$$
n P\left\{\xi: d\left(\xi_{0}, \xi\right)<\delta\right\}>1-\delta .
$$

Proof. Choose a stack $S$ of height $n$ and a semi-partition $Q^{\prime} \subset S$ such that $\rho\left(Q, Q^{\prime}\right)<\epsilon$ and $n \mu(B(S))>1-\epsilon$. The last condition can be ensured by requiring $S$ to approximate some arbitrarily chosen fine partition as well as $Q$. Note that $n$ can be chosen arbitrarily large, for the same reason. Let $\xi(x)$ denote the $Q n$-name for $x \in X$ and $\xi_{1}$ the common $Q^{\prime} n$-name of points in $B(S)$. Observe that

$$
\int_{B(S)} n d\left(\xi(x), \xi_{1}\right) d \mu x=\rho\left(Q \cap|S|, Q^{\prime}\right)<\epsilon .
$$

Thus

$$
\frac{1}{\mu(B(S))} \int_{B(S)} d\left(\xi(x), \xi_{1}\right) d \mu(x)<\frac{\epsilon}{1-\epsilon},
$$

so that we must have $d\left(\xi(x), \xi_{1}\right)<\delta$ for $x \in B(S)$ except for a set of measure at most $\delta \mu(B(S))$, where $\delta=(\epsilon /(1-\epsilon))^{1 / 2}$. Thus

$$
P\left\{\xi: d\left(\xi, \xi_{1}\right)<\delta\right\}>(1-\delta) \mu B(S) .
$$

Now $P\left(\xi_{1}\right)$ may be 0 , but if we replace $\xi_{1}$ by any string $\xi_{0}$ such that $P\left(\xi_{0}\right)>0$ and $d\left(\xi_{0}, \xi_{1}\right)<\delta$ then we have

$$
P\left\{\xi: d\left(\xi, \xi_{0}\right)<2 \delta\right\}>(1-\delta) \mu(B(S))>\frac{(1-\delta)(1-\epsilon)}{n} .
$$

A more careful choice of $\epsilon$ now completes the proof.

2.2 Construction of $\tau$. We proceed by the stacking method, using double stacks. $\tau$ will act on $(X, \mathscr{F}, \mu)$ where $X=[0,1), \mathscr{F}$ is the $\sigma$-field of Borel sets and $\mu$ is Lebesgue measure. At the $n$th stage we will have two stacks $S_{n}{ }^{0}$ and $S_{n}{ }^{1}$ whose levels are intervals of length $2^{-(n+1)}$ and $\tau$ will map each level in $S_{n}{ }^{i}$, except the top one, linearly to the level directly above. $\tau$ will be undefined on the top of $S_{n}{ }^{i}$.

We start by letting $S_{0}{ }^{0}=\left[0, \frac{1}{2}\right), S_{0}{ }^{1}=\left[\frac{1}{2}, 1\right)$. Suppose $S_{n}{ }^{0}$ and $S_{n}{ }^{1}$ have been defined and have height $2^{n}$. Cut $B\left(S_{n}{ }^{i}\right)$ into two intervals $I_{n}{ }^{i}$ and $J_{n}{ }^{i}$ of equal length, and let $\tau$ map $\tau^{2^{n}-1} I_{n}{ }^{0}$ linearly to $J_{n}{ }^{1}$ and $\tau^{2^{n}-1}\left(I_{n}{ }^{i}\right)$ linearly to $J_{n}{ }^{0}$. This defines stacks $S_{n+1}{ }^{0}$ and $S_{n+1}{ }^{1}$ of height $2^{n+1}$ over $I_{n}{ }^{0}$ and $I_{n}{ }^{1}$. In the limit $\tau$ is defined everywhere and is a measure preserving transformation. This example, constructed in this way, is due to Baxter [2].

Lemma 2.3. $\tau$ is ergodic.

This can be shown by a variation of the usual argument for ordinary stacking transformations [5, Theorem 6.2], noticing that for each $n$, half of $S_{n}{ }^{0}$ moves to $S_{n}{ }^{1}$ and vice-versa. 
Proposition 2.4. $\tau$ has simple spectrum.

Proof. Let $\sigma$ be the transformation $\sigma: x \mapsto x+\frac{1}{2}(\bmod 1)$ of $[0,1)$. It is immediate by induction that $\sigma S_{n}{ }^{0}=S_{n}{ }^{1}$ for all $n$ which implies that $\sigma$ commutes with $\tau$. Let $U_{\sigma}$ and $U_{\tau}$ be the unitary operators on $\mathscr{H}=L_{2}(X, \mathscr{F}, \mu)$ defined by $U_{\sigma} f=f \circ \sigma^{-1}$ and $U_{\tau} f=f \circ \tau^{-1}$. Then the subspaces

$$
\begin{aligned}
& \mathscr{H}_{0}=\left\{f: U_{\sigma} f=f\right\} \\
& \mathscr{H}_{1}=\mathscr{H}_{0} \perp=\left\{f: U_{\sigma} f=-f\right\}
\end{aligned}
$$

are invariant under $U_{\tau}$. Furthermore it is clear that $U_{\tau} \mid \mathscr{H}_{0}$ has discrete spectrum and its eigenvalues are all the $2^{n}$ th roots of unity, $n \in Z^{+}$. (Indeed $\tau$ acting on the $\sigma$-field of sets $E$ such that $\sigma(E)=E$ is just the famous von-NeumannKakutani transformation as described in [5, Ex. 6.4].) Since the eigenvalues are distinct, $U_{\tau} \mid \mathscr{H}_{0}$ has simple spectrum.

Let $B_{n}{ }^{i}=B\left(S_{n}{ }^{i}\right)$ and $f_{n}=\chi_{B_{n}{ }^{0}}-\chi_{B_{n}{ }^{1}} \in \mathscr{H}_{1}$. For $f \in \mathscr{H}$ denote by $\mathscr{H}(f)$ the closed linear span of $\left\{U_{\tau}{ }^{i} f: i \in Z\right\}$. Obviously $f_{n} \rightarrow 0$ and it is easy to see that $\mathscr{H}\left(f_{n}\right) \uparrow \mathscr{H}_{1}$. It follows by $\left[\mathbf{2}\right.$, Lemma 14] that $U_{\tau} \mid \mathscr{H}_{1}$ has simple spectrum.

We now show that $U_{\tau} \mid \mathscr{H}_{1}$ has continuous spectrum (i.e. no eigenvectors). Suppose, then, that there is an $f \in \mathscr{H}_{1}$ such that $U_{\tau} f=\lambda f$. Then $f^{2} \in \mathscr{H}_{0}$ and $U_{\tau} f^{2}=\lambda^{2} f^{2}$. By our analysis of $\left.U_{\tau}\right|_{\mathscr{H}_{0}}$ it follows that $\lambda^{2}$ is a dyadic root of unity and hence $\lambda$ is as well. Thus $U_{\tau}$ has two orthogonal eigenfunctions with the same eigenvector, which contradicts the ergodicity of $\tau$.

Now $\left.U_{\tau}\right|_{\mathscr{H}_{0}}$ and $\left.U_{\tau}\right|_{\mathscr{H} 1}$ can be represented as multiplication by $z$ on $L_{2}(S, \mathscr{F}$, $\left.\mu_{0}\right)$ and $L_{2}\left(S, \mathscr{F}, \mu_{1}\right)$ respectively where $S$ denotes the unit circle in the complex plane. Moreover $\mu_{0}$ and $\mu_{1}$ are mutually singular since $\left.\bar{U}_{\tau}\right|_{\mathscr{H}_{0}}$ has discrete spectrum and $\left.U_{\tau}\right|_{\mathscr{H}_{1}}$ has continuous spectrum so $U_{\tau}=\left.\left.U_{\tau}\right|_{\mathscr{H}_{1}} \oplus U_{\tau}\right|_{\mathscr{H}_{2}}$ can be represented as multiplication by $z$ on $L_{2}\left(S, \mathscr{F}, \mu_{0}+\mu_{1}\right)$ and hence has simple spectrum.

THEOREM 2.5. $\tau$ has rank 2.

Proof. $\tau$ clearly has rank at most 2, so we must show it does not have rank one. Let $Q_{0}=\left[0, \frac{1}{2}\right), Q_{1}=\left[\frac{1}{2}, 1\right)$ and let $Q=\left\{Q_{0}, Q_{1}\right\}$. We are going to show $(\tau, Q)$ violates the conclusion of Proposition 2.1. We begin by examining the structure of $Q$-names.

Let ${\sigma_{n}}^{i}, i \in\{0,1\}$, denote the common $Q-2^{n}$-name of points in $B\left(S_{n}{ }^{i}\right)$. We will call $\sigma_{n}{ }^{0}$ and $\sigma_{n}{ }^{1} n$-symbols. (The terms $n$-symbol and $n$-string should not be confused.) Clearly

$$
\sigma_{0}{ }^{0}=0, \quad \sigma_{0}{ }^{1}=1, \quad \sigma_{n+1}{ }^{0}=\sigma_{n}{ }^{0}{\sigma_{n}}^{1}, \quad \sigma_{n+1}{ }^{1}=\sigma_{n}{ }^{1} \sigma_{n}{ }^{0} .
$$

It follows that $\sigma_{n}{ }^{0}$ is the initial segment of length $2^{n}$ of the Morse minimal sequence (one-sided version). Note that $\sigma_{n}{ }^{0}$ and $\sigma_{n}{ }^{1}$ are obtained from each other by changing each 0 to a 1 and each 1 to a 0 . Observe further that $\sigma_{n+1}{ }^{0}$ can be obtained by inserting a 1 after each 0 and a 0 after each 1 in $\sigma_{n}{ }^{0}$. More generally if we replace the 0 's and 1's in $\sigma_{n}{ }^{0}$ by $\sigma_{m}{ }^{0}$ and $\sigma_{m}{ }^{1}$ respectively we get $\sigma_{m+n}{ }^{0}$. (This is easily seen by induction.) 
We now come to a lemma which is crucial to the proof of Theorem 2.5. To state it we need some terminology. If $\xi$ is an $m$-string and $\eta$ is an $r$-string such that $d(\eta, \xi[i, i+r-1]) \leqq \epsilon$ we say that $\eta$ appears within $\epsilon$ at $i$ in $\xi$. If $\xi=0$ we simply say $\eta$ appears at $i$ in $\xi$.

Lemma 2.6. Suppose $\alpha_{1}$ and $\alpha_{2}$ are $n$-symbols and $\alpha_{1} \alpha_{2}$ appears within $1 / 8$ at $i$ in $\sigma_{M}{ }^{0}$ or $\sigma_{M}{ }^{1}$. Then $i$ is a multiple of $2^{n}$ and the appearance is necessarily exact.

Proof. The proof is by induction on $n$. Note that the result is vacuous for $n=0$. Now suppose $\alpha_{1}$ and $\alpha_{2}$ are $(n+1)$-symbols so $\alpha_{j}=\beta_{j} \gamma_{j}$ where $\beta_{j}$ and $\gamma_{j}$ are opposite $n$-symbols for $j=1,2$. Suppose that $\alpha_{1} \alpha_{2}$ appears within $1 / 8$ at $i$ in $\sigma_{M}{ }^{0}$ or $\sigma_{M}{ }^{1}$. Then either $\alpha_{1}$ appears within $1 / 8$ at $i$ or $\alpha_{2}$ appears within $1 / 8$ at $i+2^{n+1}$, so by the induction hypothesis we can conclude $i=k 2^{n}$. Thus each of $\beta_{1}, \gamma_{1}, \beta_{2}, \gamma_{2}$ is matched within $1 / 2$ with the appropriate $n$-symbol in $\sigma_{m}{ }^{0}$ and hence each of them appears exactly, since the distance between $n$-symbols is either 0 or 1.

We now suppose that $k$ is odd and derive a contradiction. Since $k$ is odd, $\sigma_{M}{ }^{0}\left[(k+1) 2^{n},(k+3) 2^{n}-1\right]$ is an $(n+1)$-symbol, say $\sigma_{n+1}{ }^{0}$ for definiteness. Thus $\gamma_{1}=\sigma_{n}{ }^{0}$ and $\beta_{2}=\sigma_{n}{ }^{1}$ and it follows that $\beta_{1}=\sigma_{n}{ }^{1}$ and $\gamma_{2}=\sigma_{n}{ }^{0}$ since $\beta_{1} \gamma_{1}$ and $\beta_{2} \gamma_{2}$ are $(n+1)$-symbols. Thus

$$
\sigma_{M}{ }^{0}\left[k 2^{n},(k+4) 2^{n}-1\right]=\sigma_{n}{ }^{1} \sigma_{n}{ }^{0} \sigma_{n}{ }^{1} \sigma_{n}{ }^{0} .
$$

Recalling our remark on how $\sigma_{M}{ }^{0}$ is formed from $\sigma_{M-n}{ }^{0}$ it follows that $\sigma_{M-n}{ }^{0}[k, k+3]=1010$. Since $k$ is odd, $\sigma_{M-n}^{0}[k-1, k]$ is a 1 -symbol so $\sigma_{M-N}{ }^{0}(k-1)=0$ and similarily $\sigma_{M-n}^{0}(k+4)=1$. Thus we finally conclude that the string 000 appears in $\sigma_{M-n-1}{ }^{0}$ which is clearly impossible. This completes the proof of the lemma.

Now for each $k \in Z^{+}$, let $M_{k}$ be the maximum probability of a single $k$-string under the process $(\tau, Q)$. The following lemma provides bounds on certain $M_{k}$, the point of which will become clear at the end of the proof of Theorem 2.5.

LEMma 2.7 . For $7 \leqq k \leqq 14,(k+2) M_{k} \leqq 11 / 12$.

Proof. First we calculate the probabilities of certain strings. $P(01)$ is just $\lim f(n) / 2^{n+1}$ where $f(n)$ is the number of appearances of 01 in $\sigma_{n}{ }^{0}$ and $\sigma_{n}{ }^{1}$. Now $f_{n+1}=2 f_{n}$ unless a new 01 is formed in $\sigma_{n+1}{ }^{0}$ at the join between $\sigma_{n}{ }^{0}$ and $\sigma_{n}{ }^{1}$ which happens precisely for $n$ even. Since $f_{1}=1 / 4$ it follows that

$$
P(01)=\sum_{n=1}^{\infty}\left(\frac{1}{4}\right)^{n}=\frac{1}{3} .
$$

We then fill in the remaining 2 -string probabilities by symmetry:

(i) $P(01)=P(10)=1 / 3, \quad P(00)=P(11)=1 / 6$.

The 3 -string probabilities can be obtained in a similar manner. They are:

(ii) $P(000)=P(111)=0 \quad P(\xi)=1 / 6 \quad$ for all other $\xi$ 's. 
Now suppose $\sigma_{n}{ }^{0}$ appears in $\sigma_{M}{ }^{0}$ (or $\sigma_{M}{ }^{1}$ ) at $i$. Then $i=k 2^{n-1}$ by Lemma 2.6 and we conclude that 01 appears at $k$ in $\sigma_{M-n+1}{ }^{0}$. It follows that

(iii) $P\left(\sigma_{n}{ }^{0}\right)=P\left(\sigma_{n}{ }^{1}\right)=2^{n-1} / 3$.

We now turn to $M_{k}, 7 \leqq k \leqq 14$. If $\xi$ is a 7 -string then there must appear in $\xi$ a string of the form $\alpha \beta \gamma$ where $\alpha, \beta$ and $\gamma$ are 1 -symbols. It follows by the argument used to show (iii) that

$$
P(\xi) \leqq P(\alpha \beta \gamma) \leqq \frac{1}{2} M_{3} .
$$

Since $M_{3}=1 / 6$ by (ii) we have $M_{7} \leqq 1 / 12$ and a fortiori $M_{8}, M_{9} \leqq 1 / 12$.

Now suppose $\xi$ is a 10 -string. Suppose that a string $\alpha \beta, \alpha$ and $\beta 2$-symbols, appears in $\xi$. Then if $\xi$ appears in $\sigma_{M}^{j}, \alpha \beta$ appears at $4 k$ by Lemma 2.6. But then either $\sigma_{M}{ }^{j}[4 k-4,4 k-1]$ or $\sigma_{M}{ }^{j}[4 k+8,4 k+11]$ is determined since they are 2 -symbols and $\xi$ extends into at least one of them. Thus we conclude that $P(\xi) \leqq \frac{1}{4} M_{3}=1 / 24$.

Now suppose that only one full 2 -symbol $\beta$ appears in $\xi$. Then $\xi$ has the form $\alpha \beta \gamma$ where $\alpha$ (respectively $\gamma$ ) is a 3 -string ending (respectively beginning) a 2 -symbol. We claim that if $\xi$ appears at $i$ in $\sigma_{M}{ }^{0}$ then $i+3$ is a multiple of 4 . Indeed if this is not the case then $i+5=4 k$ and we have the following picture, assuming $\gamma=0110$ for definiteness.

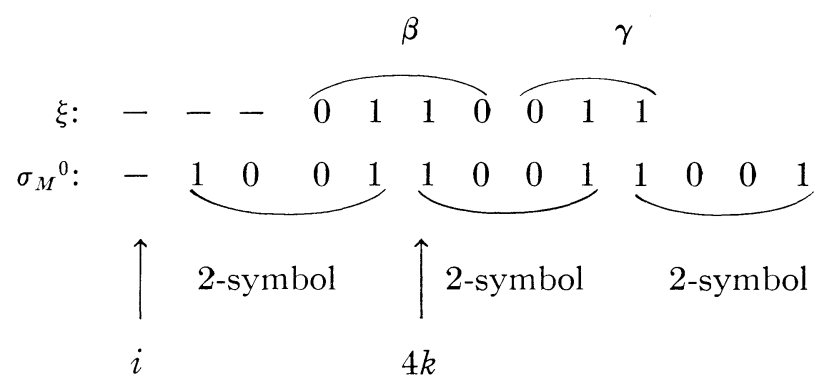

Thus we would have the string 111 occurring in $\sigma_{M-2}{ }^{0}$ which is impossible. Thus $i+3$ is a multiple of 4 . But then the 2 -symbols $\sigma_{M}{ }^{0}[i-1, i+2]$, $\sigma_{M}^{0}[i+3, i+6]$ and $\sigma_{M}{ }^{0}[i+7, i+10]$ are all determined by $\alpha, \beta$ and $\gamma$ so we conclude that $P(\xi) \leqq M_{3} / 4=1 / 24$. Thus we have $M_{10} \leqq 1 / 24$ and this takes care of $M_{11}$ through $M_{14}$ also.

Remark. The argument we gave for $M_{10}$ can also be made to work for $M_{9}$, showing that already $M_{9} \leqq 1 / 24$.

We are now ready to complete the proof of Theorem 2.5, using Proposition 2.1. We suppose, then, that $\xi$ is an $n$-string, $n \geqq 8$, such that $P(\xi)>0$ and we shall show that

$$
n P\{\eta: d(\xi, \eta),<1 / 12\} \leqq 11 / 12 .
$$


Denoting by $f(\xi, M)$ the number of $1 / 12$-appearances of $\xi$ in $\sigma_{M}{ }^{0}$ and $\sigma_{M}{ }^{1}$, what we have to show is that

$$
\frac{n}{2^{M+1}} f(\xi, M) \leqq \frac{11}{12}
$$

for all $M$.

Let $k$ and $r$ be such that

$$
k 2^{r} \leqq n<(k+1) 2^{r}, \quad 8 \leqq k \leqq 15 .
$$

Since $P(\xi)>0, \xi$ must appear in $\sigma_{L}^{0}$ for some $L$. Since $\sigma_{L}{ }^{0}$ is a concatenation of $r$-symbols it follows that there is a string of at least $k-1 r$-symbols, say $\alpha_{1} \ldots \alpha_{k-1}$, appearing at an $i$ in $\xi$. If $\xi$ appears within $1 / 12$ at $j$ in $\sigma_{M}{ }^{0}$ (or $\sigma_{M}{ }^{1}$ ) then $\alpha_{1} \ldots \alpha_{k-1}$ appears within $(8 / 7)(1 / 12)$ at $j+i$, hence by Lemma 2.5 , $j+i$ is a multiple of $2^{r}$ and $\alpha_{1} \ldots \alpha_{k-1}$ appears exactly. Thus by the usual argument

$$
\overline{2}^{\frac{1}{M+1}} f(\xi, M) \leqq P\left(\alpha_{1} \ldots \alpha_{k-1}\right) \leqq \frac{1}{2^{r}} M_{k-1} .
$$

But then we have

$$
\overline{2}^{\frac{n}{M+1}} f(\xi, M) \leqq \frac{n}{2^{r}} M_{k-1} \leqq(k+1)\left(M_{k-1}\right) \leqq \frac{11}{12}
$$

by Lemma 2.6. This completes the proof.

We will now indicate why $\tau$ is isomorphic to the transformation generated by the Morse minimal sequence, which we call $T$. $T$ can be characterized abstractly as the shift transformation on $\{0,1\}^{z}$ restricted to the orbit closure $\overline{\mathscr{O}}$ of the two-sided Morse minimal sequence, the measure $\mu$ being the unique $T$-invariant and $T$-ergodic probability measure on $\overline{\mathscr{O}}$. A proof that $T$ and $\tau$ are isomorphic can be based on these facts alone. However we will use the explicit definition of $\mu$, namely that its value on finite strings is the frequency of appearance of that string in the Morse minimal sequence (one-sided or two-sided). Now the partition $Q$ sets up an isomorphism between $\tau$ acting on $\bigvee_{i=-\infty}^{\infty} \tau^{i} Q$ and the shift on $\{0,1\}^{Z}$ with the measure we have called $P$, and as we have already seen the value of $P$ on finite strings is obtained by taking the limiting frequency of appearance in $\sigma_{n}{ }^{0}$ and $\sigma_{n}{ }^{1}$. But since ${\sigma_{n+1}}^{0}={\sigma_{n}}^{0}{\sigma_{n}}^{1}$ it is easily seen that this is the same as the limiting frequency in $\sigma_{n}{ }^{0}$ or in other words, the actual frequency in the one-sided Morse minimal sequence. Thus $\tau$ on $\bigvee_{i=-\infty}^{\infty} \tau^{i} Q$ is osomorphic to $T$. We'd like to know that $\bigvee_{i=-\infty}^{\infty} \tau^{i} Q$ is the full $\sigma$-field of Borel sets on $[0,1)$. To see this it suffices to show that for almost all $x \in[0,1)$, the full two-sided $Q$-name of $x$ determines $x$. This in turn follows quite easily from Lemma 2.6 , in fact from a weaker version of Lemma 2.6 with $1 / 8$-appearances replaced by exact appearances. 
3. Characterization of rank one. Let $\xi$ be an $r$-string and $\delta>0$. An $n$-string $\eta$ will be called a $\delta-\xi$-string if it has the form

$$
\epsilon_{0} \xi_{1} \epsilon_{1} \xi_{2} \ldots \xi_{l} \epsilon_{l}
$$

where the $\epsilon_{i}$ are strings subject only to the condition $\sum_{i=0}^{l}\left|\epsilon_{i}\right|<\delta$.

THEOREM 3.1. $\tau$ has rank one if and only if for each partition $Q$ and $\delta>0$ there is an $N$ and an $r$-string $\xi$ such that for $n>N$, with probability $1-\delta$ an $n$-string agrees with a $\delta-\xi$-string within $\delta$.

Proof. Suppose $\tau$ has rank one and $Q$ is a partition. Then by the proof of Proposition 2.1 there is a stack $S$ of height $r$, a set $G \subset B(S)$ and an $r$-string $\xi$ such that $\mu|S|>1-\epsilon, \mu(G)>(1-\epsilon) \mu B(S)$ and the $Q$ - $n$-name of any point in $G$ is within $\epsilon$ of $\xi$. Now by the ergodic theorem, for $n$ sufficiently large there is a set $\bar{G}$ such that for $x \in \bar{G}$

$$
\frac{1}{n} \#\left\{i: 0 \leqq i<n, \tau^{i} x \in \bigcup_{j=0}^{i-1} \tau^{j} G\right\}>1-3 \epsilon .
$$

It now follows easily that the $n$-name of any $x \in \bar{G}$ is a $\delta-\xi$-string within $\delta$ for a suitable choice of $\epsilon$.

Now suppose that $\tau$ satisfies the string condition and $Q$ is any partition. Note that $\tau$ is necessarily ergodic. Choose an $n$, an $r$-string $\xi$ and a set $G \subset X$, $\mu(G)>1-\epsilon$, such that for $x \in G$ the $n$-name of $x$ is within $\epsilon$ of an $\epsilon-\xi$ string. By a well-known variant of Rohlin's lemma [6, p. 71, Lemma 2] we may now find a set $B \subset G$ such that $B, \tau B, \ldots, \tau^{n-1} B$ are disjoint and

(i) $\mu \bigcup_{i=0}^{n-1} \tau^{i} B>1-2 \epsilon$.

For $x \in B$ let $\eta(x)$ denote the $Q$-n-name of $x$ and let $\bar{\eta}(x)$ be an $\epsilon-\xi$-string such that $d(\eta(x), \bar{\eta}(x))<\epsilon$. Now define a partition $\bar{Q}$ of $\bigcup_{i=0}^{n-1} \tau^{i} B$ by decreeing that the $\bar{Q}-n$-name of each $x \in B$ is $\bar{\eta}(x)$. As in the proof of proposition 2.1 we have

(ii) $\rho\left(Q \cap \bigcup_{i=0}^{n-1} \tau^{i} B, \bar{Q}\right)<\epsilon$.

Now we define a $\tau$ stack $S$ as follows. Let $B_{\bar{\eta}}=\{x \in B: \bar{\eta}(x)=\bar{\eta}\}$. Since $\bar{\eta}$ is an $\epsilon-\xi$-string there are integers $i(1), \ldots, i(l)$ such that $l r \geqq(1-\epsilon) n$, $i(j)+r \leqq i(j+1)$ and $\bar{\eta}[i(j), i(j)+r-1]=\xi$. We let

$$
A_{\bar{\eta}}=\bigcup_{j=1}^{l} \tau^{i(j)} B_{\bar{\eta}} \text { and } A=\bigcup_{\bar{\eta}} A_{\bar{n}} .
$$

Then $A$ is clearly the base for a $\tau$-stack $S$ of height $r$ such that $|S| \subset \cup_{i=0}^{n-1} \tau^{i} B$ and

$$
\text { (iii) } \mu|S|>(1-\epsilon) \mu \bigcup_{i=0}^{n-1} \tau^{\imath} B \text {. }
$$


Furthermore the $\bar{Q}-n$-name of any point in $A$ is $\xi$ which means that $\bar{Q} \cap|S| \subset S$. Since (i) (ii) and (iii) imply that $\rho(Q, \bar{Q} \cap|S|)$ is small we have shown that the partition $Q$ can be approximated by a subpartition of a stack so $\tau$ is rank 1 , as desired.

Corollary 3.2. A factor of a rank one transformation is rank one.

Remark. Theorem 3.1 may be extended in a fairly straightforward way to transformations of rank $\leqq n$. Thus Corollary 3.2 holds also for rank $\leqq n$. (Obviously rank may decrease on passing to a factor.)

\section{REFERENCES}

1. J. R. Baxter, A class of ergodic automorphisms, Thesis, University of Toronto, 1969.

2. - A class of ergodic transformations having simple spectrum, Proc. Amer. Math. Soc. 24 (1970).

3. R. V. Chacon, Weakly mixing transformations which are not strongly mixing, Proc. Amer. Math. Soc. 22 (1969), 559-562.

4. Approximation and spectral multiplicity, Contributions to Ergodic Theory and Probability, Springer Lecture Notes 160 (1970), 18-27.

5. N. A. Friedman, Introduction to ergodic theory (Van Nostrand Reinhold, 1970).

6. P. R. Halmos, Ergodic theory (Chelsea, 1956).

7. A. del Junco, Transformations with discrete spectrum are stacking transformations, Can. J. Math. 28 (1976), 836-839.

8. S. Kakutani, On the ergodic theory of shift transformations, Proc. 5th Berkeley Symp. 2 part ii, 405-414. (University of Calif. Press, Berkeley, 1967).

9. - Strictly ergodic symbolic dynamical systems, Proc. 6th Berkeley Symp. 2, 319-326 (University of Calif. Press, Berkeley, 1972).

10. B. Weiss, Equivalence of measure preserving transformations, preprint.

University of British Columbia, Vancouver, British Columbia 